\title{
Urban concept of a healthy city: case study - Tripoli, Libya
}

\author{
T. Amer \\ Dept. of Architecture and Urban Planning, Faculty of Engineering, \\ Tripoli University, Libya
}

\begin{abstract}
At a time when there is a close relationship between the occurrence of many types of diseases and a city urban environment it appears that a more positive approach is needed for the integration of urban development and healthy city issues in urbanized areas. It is against this background that the Healthy City project was established in order to consider ways in which health-based approaches can take place in our new cities' planning policies. This research, which is based on the proceeding of such concepts will discuss the role of the urban planning process in the improving of urban environmental qualities in Tripoli city as a case study. Tripoli city accommodates more than $35 \%$ of the total country's population and which, by now, can be regarded as almost the antinomy of such a concept. In this respect, architects and urban planners will play a substantial role in the trend toward the improvement of environmental quality which in consequence would lead to undertakings to protect people from the hazards of environmental diseases. According to world tendencies oriented towards creating a healthy city, this research will be an attempt to evaluate the prospective of the urban concept of a healthy city in subtropical climate zones based on physical, social, and psychological environment supportive to health, keeping in mind the area's local conditions (topographic, social and culture). Keywords: healthy city, ecological city, psychological environment, diseases, health-based concepts, anti-pedestrianism, maximum variety, close system, minimal intrusion.
\end{abstract}

\section{Introduction}

Many health organizations and public health units all over the world recognized the significance of the impacts of urban environmental conditions on human 
health. It was discovered that close relationships exist between these conditions and the occurrence of various types of illnesses and diseases. Such a situation is typical, especially for many cities and it comes as a result of the processes of the demographic and the consequent dynamics of urban development. The two mentioned processes are the most significant features of contemporary cities and their urban civilization. It must the underlined that the said situation is much more unfavourable in the cities of developing countries located in sub-tropical and tropical zones, where the rapid growth of cities and urban areas is faster, particularly in the large cities where the administration services, economic growth and social services are over concentrated. This has caused extensive migration from small and medium-size towns which offer less job opportunities, less social needs and less availability of infrastructure.

"In developing countries, the deterioration of the environment has a very wide range of causes and effects; problems vary from a global perspective, (e.g. ozone depletion) through international, national and regional perspectives to localized; one such as litter on the streets." This quoted statement by Classen [1], emphasized the fact that these cities have their own environmental problems with different characteristics depending upon a series of manifold factors such as the local conditions.

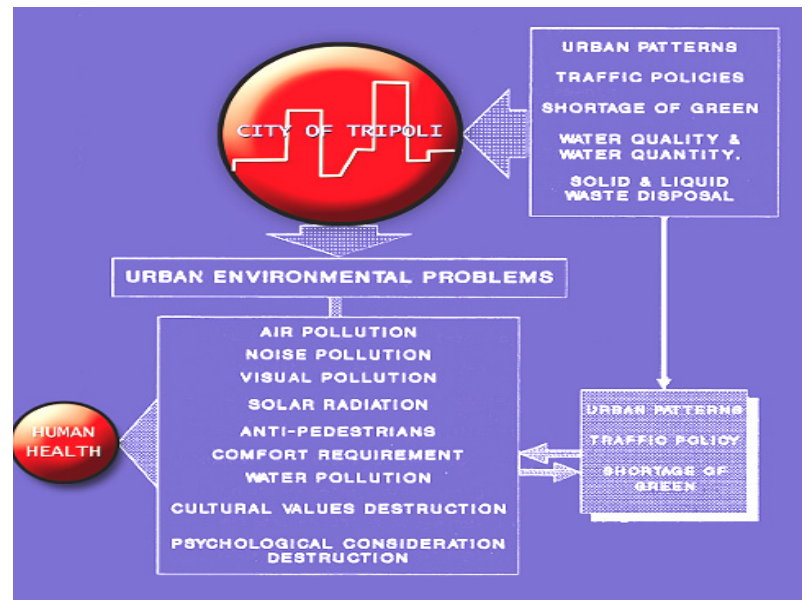

Figure 1: $\quad$ Main factors behind environmental problems (Tripoli city).

The above scheme (figure 1) points out some of the most important features distinguishable in the cities of developing countries. A schematic presentation of the manifold aspects of these cities: the upper part of the diagram outlines the essential issues which affect the overall condition of life in a developing city (e.g. the policies of urban development, urban transportation policies, housing policies, economical policies, etc.). These are the main subjects to be taken into account in order to avoid and/or lessen the ecological threats related to the urbanized areas. The lower part of the scheme shows the major ecological threats on human health resulting from the above mentioned policies, (e.g. 
overpopulation, pollution and noise aspects, green open spaces destruction, etc). These are considered to be the main causes of the spread of many illnesses and diseases.

\section{The urban environment and health hazards}

The basic problem of our times found their reflection in the shaping of spaces around us. Industrialization and the rapid development of cities created certain urban environment problems which transformed our cities into unhealthy areas (Curtis [2]). The city environment is increasingly pathological, both in terms of the inhabitants' health, the state of the natural environment and the urbanized areas. The governments and societies of all countries in the world are more and more concerned with the spread of diseases, illnesses and disorders related to the city environment. An increasing number of disorders of a general nature are undetermined and difficult to localize, such as discomfort, general feeling of ill health, weakness and ponderousness, dizziness, stupefaction, difficulty with concentration, somnolence during the day time, insomnia at night and state of anxiety are currently being observed in various phenomena. These clinical diagnoses were recognized as being linked to environmental conditions. In relation to this subject (WHO [3]). Figure 2 shows how the close relationships between diseases and the city urban environment occur.

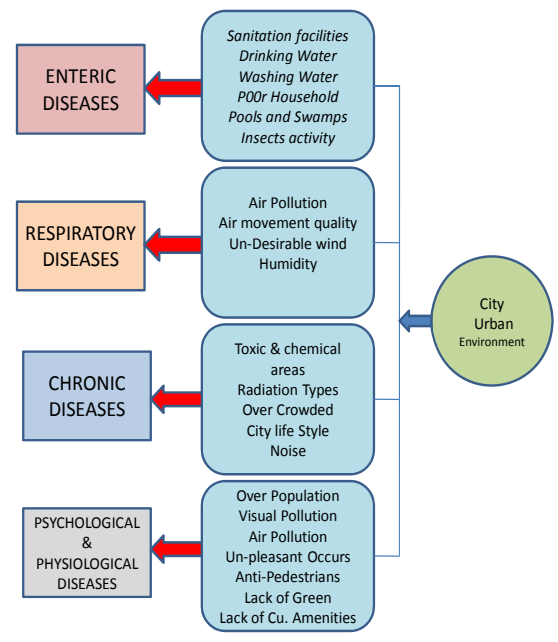

Figure 2: Close relationships between diseases and the city urban environment.

\section{Health-based concepts oriented towards the improvement of the environment}

Being directed by the imperative to sustain human health and life, which is the strongest motive of all, several extensive projects known under the name of 
Healthy City, have been undertaken. The concept of Healthy City is an active project which works on promoting the creation of a framework of health conditions in urban areas. This concept comes to life under the auspices of the WHO 1979. However, the most important aims of the healthy city concept are (WHO [4]):

- The aim to integrate city life with its health, ecological, cultural and economic issues.

- Activating all activities with respect to public health in order to fully understand the relationships existing within man's environment.

- Building-up social and physical environments supportive to health.

- The development of the ecological city model.

At the same time, the environmentalist movement promotes new approaches summarized by such key words as Self Reliance, Sustainable Development, Population Control, etc.

Assumption of the Healthy City project is relatively new and they concern mainly the problems of the cities of Europe and North America. The subject of this paper deals with a city located in the subtropical zones, as this city is very unique in terms of history and culture as an Islamic city, and have their own concept of health development. We may assume that this concept is valid for such areas but obviously demand some sort of interpretation to be fulfilled in order to express it in the form of workable solutions in a different case study like Tripoli city.

Therefore this study is based on the following principles:

- Town planning patterns governed by ecological principles; this rule is a basic criteria of urban planning.

- Preservation and integration of traditional settlement patterns and their history as an aspiration to create new urban areas together with the new technology of urban development.

- The necessity to involve and reflect man's biological and mental needs in the urban process.

\section{Case study: environmental health issues in existing urban areas (Tripoli city)}

In order to create a firm and adequate basis for evaluation of the Tripoli urban area, the existing land use and other relative data were analysed based on the assumption and general principles of the healthy city concept. In summarizing the present environmental problems of the Tripoli urban context, the following can be stated (Tripoli Master Plan [5]).

\subsection{Overpopulation}

Tripoli's attractiveness as a prime city has recently become the main reason for rapid population growth, fast industrial, technological and territorial development (NPP [6]). This is what makes Tripoli an overpopulated city compared to other Libyan cities. 

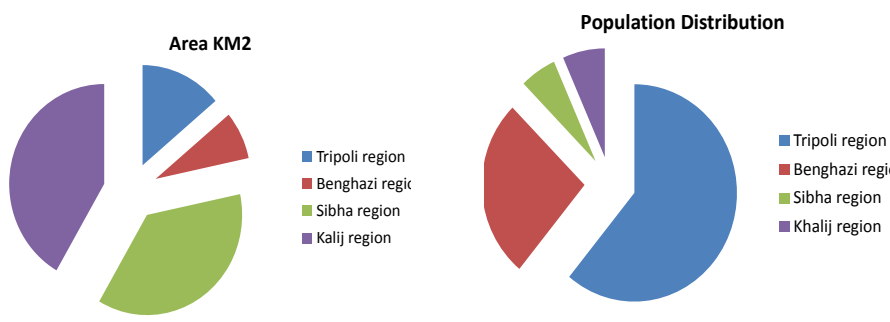

Figure 3: $\quad$ Population compared to the area of the main region.

\subsection{Air pollution}

The main sources of air pollution are:

- Unstable sand dunes in south areas covering more than $16 \%$ of the total area of Tripoli agglomeration.

- Transportation facilities (Tripoli harbour, disorganized public transportation, high traffic flow and congestion points.

- Inadequate garbage collection system.

- Discharging unprocessed sewage from the city to adjacent areas mainly to sea water.

\subsection{Solar radiation}

Recent urban is associated with the western urbanization model characterized by wide highways and raised buildings with open plan design, which maximize the solar radiation in urban areas. Traffic areas like wide streets and huge parking places are considered to be one causes of radiation, together with the lack of green open spaces which maximize the radiation problem in Tripoli urban areas.
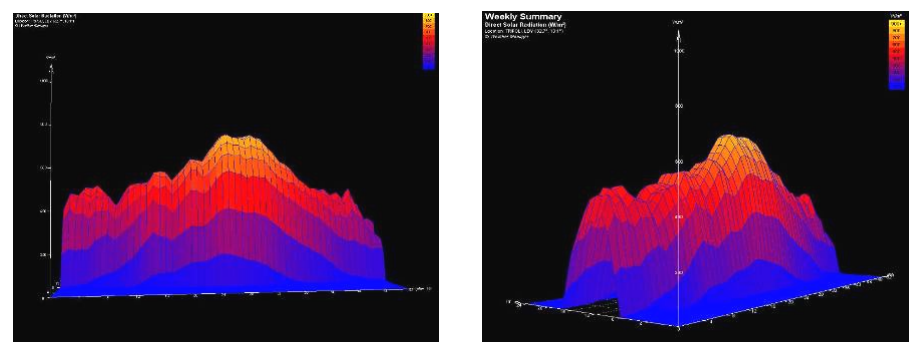

Figure 4: $\quad$ Annual and weekly direct solar radiation Tripoli.

\subsection{Anti-pedestrianism}

Pedestrians are the most hindered and endangered class of all the traffic participants in Tripoli with lack of protection elements from the sun.

The main reason being the sidewalks are almost everywhere either too narrow, unpaved, used for car parking or full of obstacles. Lack of traffic-free 
zones for pedestrians particularly in the central area of the city where the most serious problems for pedestrians occur and where the heaviest movement were observed.

\subsection{Shortage of green areas}

The green areas in Tripoli urban areas have been mostly destroyed by urban expansion among many other factors such water scarcity, sand movement, etc.

- The existing green area amounts to only $3-4 \mathrm{~m}^{2} / \mathrm{p}$ in Tripoli city.

- The existing green belt in the south area of Tripoli has been destroyed by unplanned urban growth.

\subsection{Air movement quality}

- Unplanned urban expansion, lacking choice of site criteria, causes poor quality of air movement between the urban elements within the city context.

- Lack of orientation consideration according to the prevailing winds in contemporary urban areas.

\section{A new urban environmental policy guideline for the city of Tripoli, based on a healthy city concept}

According to the scope of this study which emphasises the role of urban planners in the improvement of health issues in urbanized areas within the context of Tripoli city, the following set of guidelines of urban policies are recommended.

\subsection{City growth management with environmental standards}

The growth of the city has to be balanced with the improvement of an environmental standards growth management policy

\subsection{Urban planning decision based on the environmental system analysis}

To optimize environmental decisions, urban planning development should be based on environmental system analysis involving an inventory of available data for ecology, social, economy and land resources (Stover [7]).

\subsection{Urban planning process based on the ecological principles}

Apply the approach characteristics of an ecological city model with four main principles (Giroult [8]).

\subsubsection{Maximum variety}

The city should offer jobs within a large variety of industrial or commercial activities. In relation to the physical environment, the criteria of maximum variety means that working places should not be systematically separated from housing areas (figure 6). 


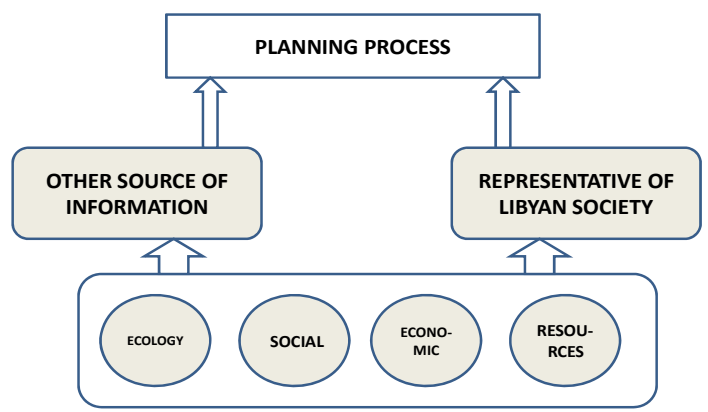

Figure 5: Environmental system analysis data base inventory - diagram.

\section{The Maximum Variety Criteria}

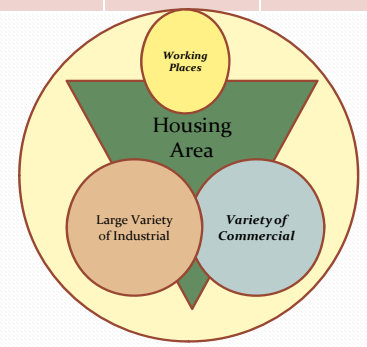

Figure 6: The maximum variety criteria and the city's main activities.

\subsubsection{Minimum intrusion}

Siting of building and urban areas or new settlements should be implemented with due consideration to the topographical, hydrographical, vegetation and climatic conditions (figure 7).

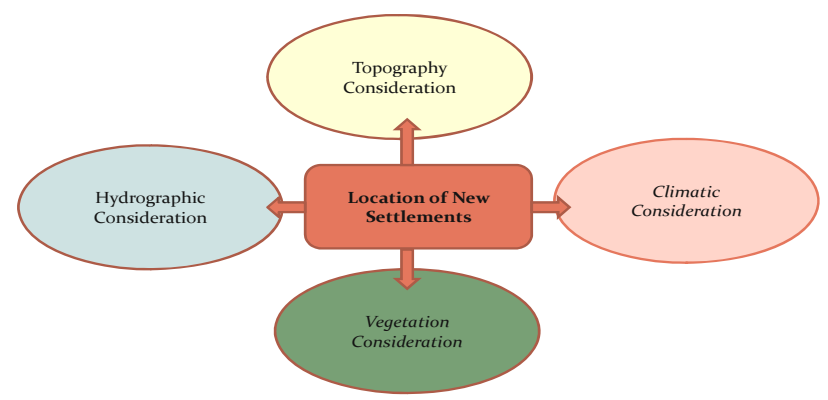

Figure 7: Minimal intrusion criteria as a basic for new settlements. 


\subsubsection{Principle of a close system}

Urban planning means, for example, that green spaces should be made available within the city. The water cycle and waste recycle should also be as close as possible within city limits (figure 8).
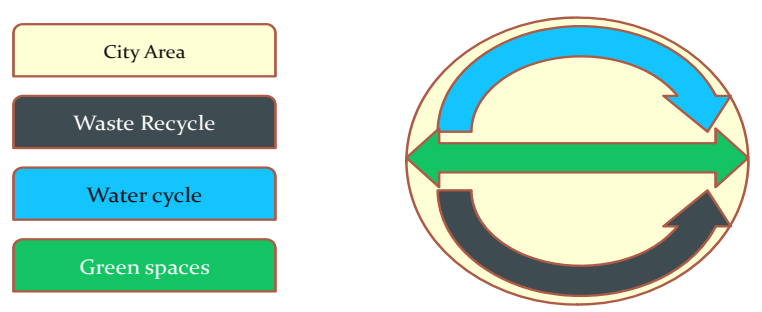

Figure 8: $\quad$ Close system principle and city planning.

\subsubsection{The optimum balance}

For each city there is an optimal size of population for which the city can provide a maximal amount of amenities, economic, and cultural opportunities, as well as housing and physical environment of high quality. This which ensure the sustainable concept of the urban development of Tripoli city.

\subsection{City restructuring incorporating the social, psychological and physical environment supportive to health}

The improvement of the physical structure of the city plays a significant role in the upgrading of its environmental quality. In the case of Tripoli, priority should be given to:

\subsubsection{Social and psychological environment supportive to health}

The physical environment of Arabic-Muslim cities is part of the tradition of Arabic societies. It is where their society, culture and religion developed and grows (Bakamur [9]). Since urban spaces represent most of this environment, a critical understanding of the concept of traditional urban patterns is therefore necessary if we are to arrive at principles which could guide the design and planning of new urban patterns.

- The concern of privacy within was reflected in the physical forms in many planning aspects in Islamic cities (courtyards courts, building heights and building openings); these aspects should be developed and considered in current urban planning policies.

- Spaces in which social integration can take place need to be provided in the existing and new urban areas. In Tripoli cultural centres, small public squares and more recreation areas should be provided in existing urban areas to encourage social integration. 


\section{Amenities, Economic, Culture, Housing and Physical Env.}

\section{Size Of Population}

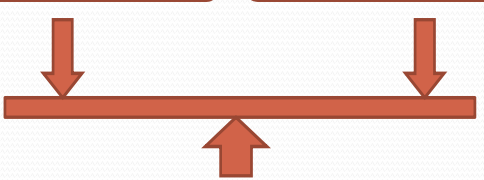

\section{Geographic Characteristics of the}

City Site

Figure 9: Optimal balance criteria as an approach to sustainable development.

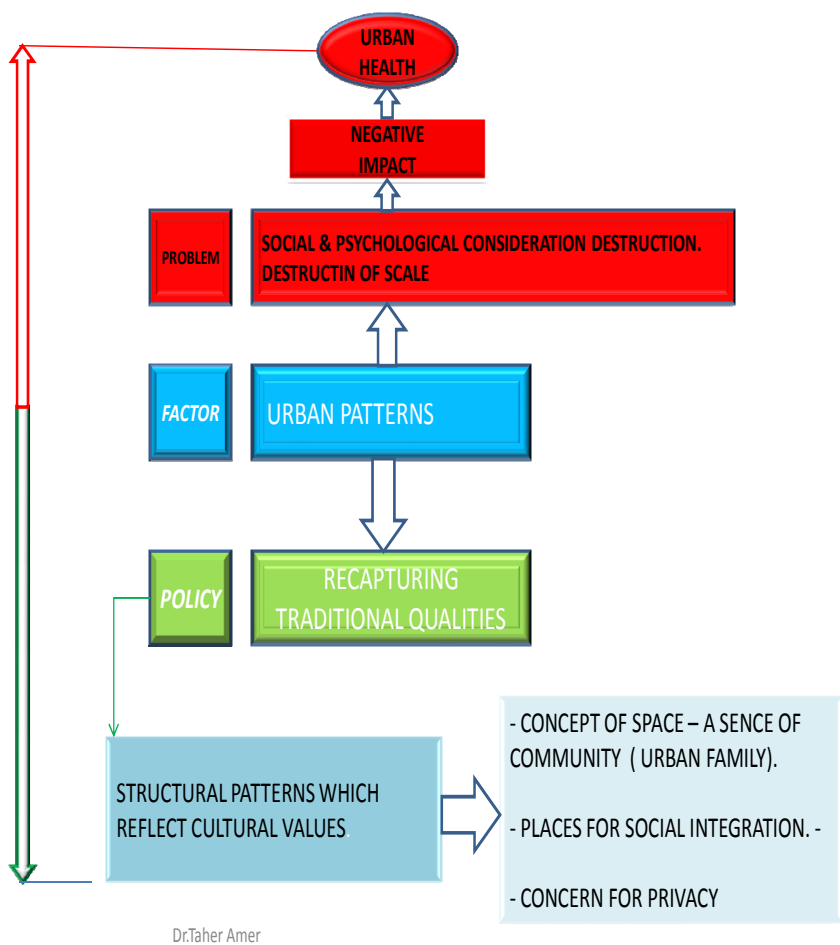

Dr.Taher Amer

Figure 10: Urban patterns, new health-based policies. 


\subsubsection{Physical environment supportive to health}

5.4.2.1 Traffic and its function elements As an essential factor in the improvement of the urban environment in Tripoli's urban area, it is recommended that environmentally sensitive traffic policies should meet the following goals.

Decrease traffic volume:

- Reducing excessive trips by improvement to the city structure and its land uses.

- More attention should be given to public transport in order to decrease traffic congestion.

- Street network differentiation to prevent through-going traffic on local streets.

- Reducing traffic in special areas - to create traffic free-zones as special areas for pedestrians.

- New modes of movement, which lie in the developing mode of movement alternative to automobiles and especially in expanding possibilities for nonmotorized movement.

- New parking spaces should be arranged in parking garages and parking lots, accommodating vehicles, particularly on the borders of central areas and pedestrian precincts.

- Developing of a cross-section of special areas.

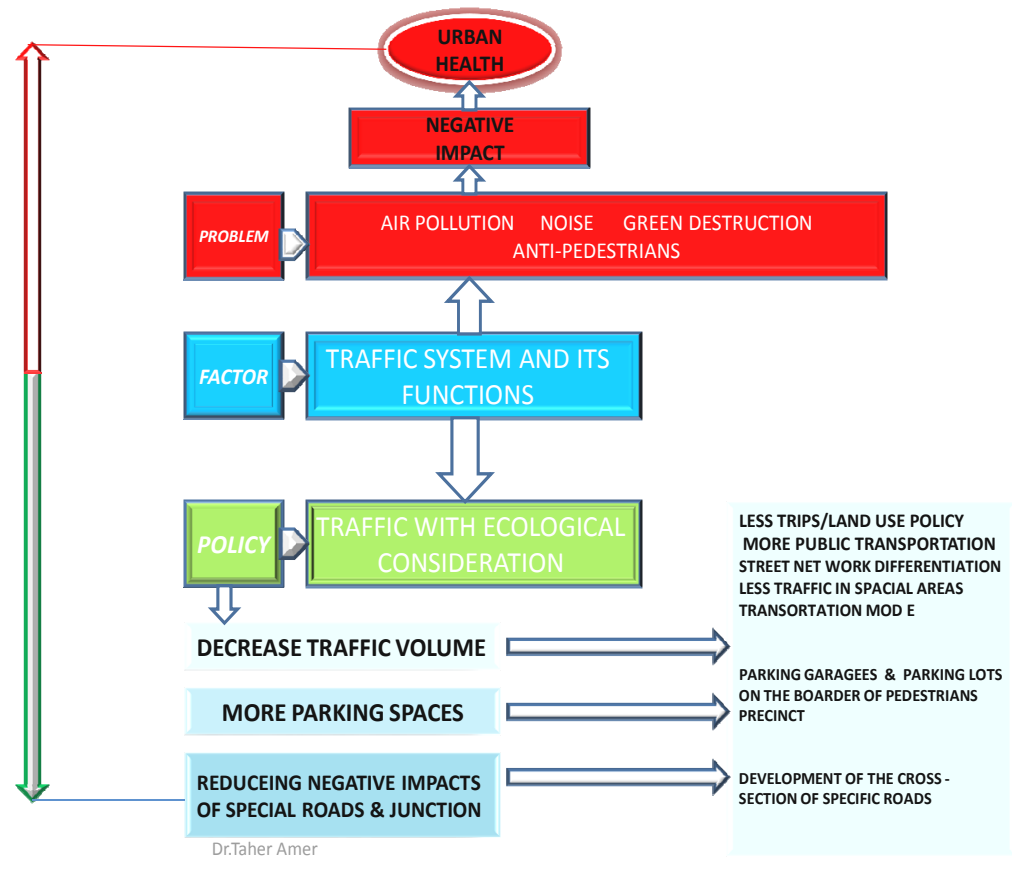

Figure 11: Factor of traffic system: health-based new policies. 


\subsubsection{Landscaping and provision of new green areas}

- Existing green areas improvement. Priority should be given to the existing areas which do not function under use such as (existing agriculture areas, existing green belt, and existing parks and gardens).

- Provision of new green areas. In this respect, green patterns should be classified and planned to cope with the local climatic conditions and water scarcity (courtyards green, small gardens, self-reliant gardens, street green, special corridors and green belts, are the main classified green areas suggested for Tripoli city).

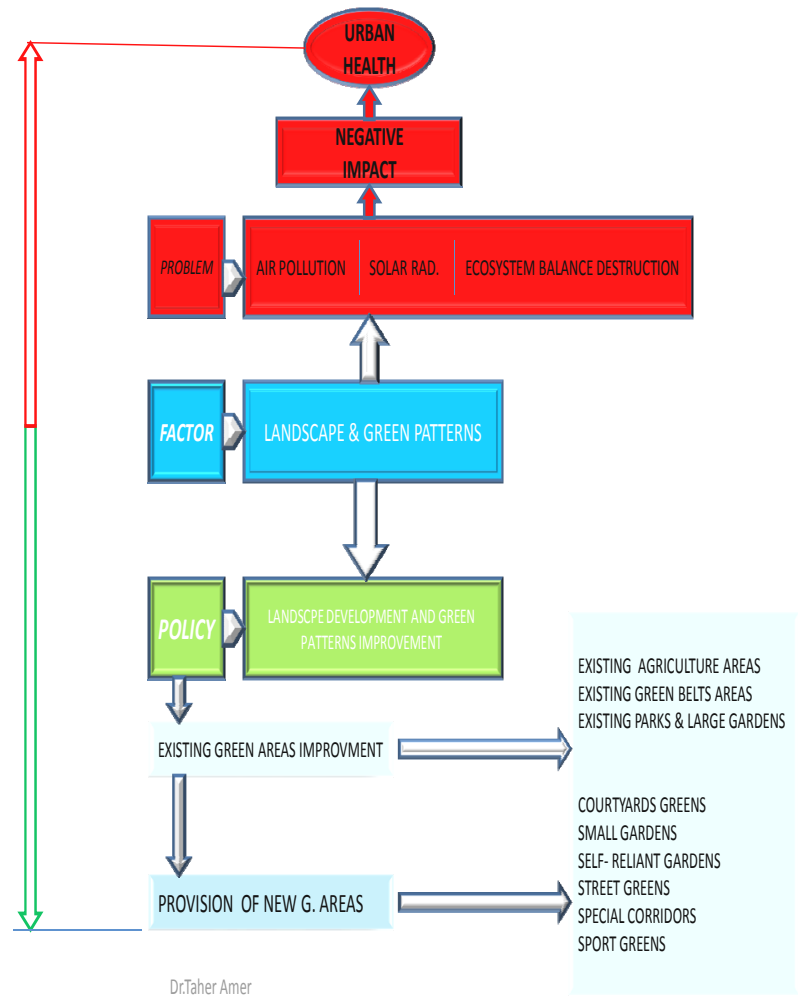

Figure 12: Landscape factor and new health-based policies.

I hope that the contents of this paper will add to our understanding of the conceptual basis of health concept in urban planning patterns. It is upon such an understanding and insight that we can gradually develop even better methods for urban planning and design and finally provide more healthy spaces and improve all environmental aspects supportive to health. 


\section{References}

[1] Dr. Claassen P. E., - Dept. of City Planning of the University of Stellenbosch - "The environment and the city" - Warsaw, Poland 25-30-81990, pp. 1-2.

[2] Curtis T. The environmental impacts of urbanization in developing world National Perspective Physical Plan UN.C for human settlements Libya 2006.

[3] WHO report - Mental health and deviance in inner cities - Geneva - 1991 pp. 3-4.

[4] Ecological models for healthy cities planning report WHO workshop, Liverpool March 1988.

[5] Tripoli Master Plan - Final report - polisercise - 1985-2000.

[6] National Perspective Physical Plan (NPP) UNC for human settlements Libya 2006.

[7] Dr. Stover L.X. Envirodynamic booklet (ESA) Phases 1, 1991.

[8] Giroult E. - Sustainable urban development, WHO Regional Office of Europe pp. 2-3.

[9] Bukamur M, Uno Arizona, Design guidelines for housing in Libya pp. 1001011985. 